\title{
Efeito do treinamento em circuito orientado para tarefa sobre o desempenho motor e a qualidade de vida de pacientes com doença de Parkinson: um estudo de caso
}

\section{Effect of task-oriented circuit training in motor performance and quality of life of patients with Parkinson's disease: a case study}

\author{
Juliana Ansaloni Chagas Pereira ${ }^{1}$, Thaís Peixoto Gaiad ${ }^{2}$, Ana Paula Santos ${ }^{3}$ \\ http://dx.doi.org/10.11606/issn.2238-6149.v27i3p329-335
}

\begin{abstract}
Pereira JAC, Gaiad TP, Santos AP. Efeito do treinamento em circuito orientado para tarefa sobre o desempenho motor e a qualidade de vida de pacientes com doença de Parkinson: um estudo de casos. Rev Ter Ocup Univ São Paulo. 2016 set.dez.;27(3):329-35.
\end{abstract}

RESUMO: Este estudo trata da descrição de dois casos de pacientes com doença de Parkinson (DP) submetidos a um programa de reabilitação diferencial, composto de treinamento em circuito orientado para tarefa. O objetivo foi analisar os possíveis benefícios do tratamento sobre o desempenho motor e a qualidade de vida em indivíduos com DP. O circuito foi constituído por cinco estações direcionadas à funcionalidade, equilíbrio e destreza manual. Foi desenvolvido durante seis semanas. Para a avaliação foram utilizados os testes de Romberg sensibilizado, velocidade da marcha e Timed Up \& Go; o questionário PDQ-39 para avaliar a qualidade de vida e a escala unificada para a avaliação da doença de Parkinson (UPDRS). O tempo gasto em cada estação e o tempo total para completar o circuito foi cronometrado nas doze sessões para análise do desempenho dos pacientes. Os dois pacientes apresentaram melhora em todos os testes avaliados, na pontuação do PDQ-39 e na escala UPDRS, além disso, houve melhora no desempenho motor em todas as estações propostas, observada através da qualidade da execução da atividade e da diminuição do tempo para executá-la. O tratamento utilizando circuito pode ser benéfico para pacientes com DP.

DESCRITORES: Doença de Parkinson/reabilitação; Qualidade de Vida; Atividade Motora
Pereira JAC, Gaiad TP, Santos AP. Effect of task-oriented circuit training in motor performance and quality of life of patients with Parkinson's disease: a case study. Rev Ter Ocup Univ São Paulo. 2016 Sept.-Dec.;27(3):329-35.

ABSTRACT: This study deals with the description of two cases of patients with Parkinson's disease (PD) submitted to a differential rehabilitation program compound of taskoriented circuit training. The aim was to analyze the benefits of treatment on motor performance and quality of life in individuals with PD. The circuit consisted of five stations aimed at functionality, balance and manual dexterity. It was developed for six weeks. For the assessment was used Romberg tests sensitized, gait speed and Timed Up \& Go; PDQ-39 questionnaire to assess quality of life and the unified Parkinson's disease rating scale (UPDRS) to assess the symptoms of PD were performed before and after treatment. The time spent in each stations and the total time to complete the circuit was timed in twelve sessions for analysis of the performance of patients. Both patients showed improvement in all evaluated tests, the score of the PDQ-39 and UPDRS, moreover, there was an improvement in motor performance in all proposed stations observed by the activity execution quality and decreasing of time to run it. The treatment using the circuit can be beneficial for patients with PD.

KEYWORDS: Parkinson Disease/rehabilitation; Quality of Life; Motor Activity.

Apresentado na III Semana de Integração do Ensino, Pesquisa e Extensão (SINTEGRA) Diamantina/ MG (2014).

1. Discente do Curso de Fisioterapia da Universidade Federal dos Vales do Jequitinhonha e Mucuri (UFVJM), Diamantina (MG), Brasil.

2. Professora Adjunta do Curso de Fisioterapia da Universidade Federal dos Vales do Jequitinhonha e Mucuri (UFVJM), Diamantina (MG), Brasil.

Endereço para correspondência: Clínica Escola de Fisioterapia, Campus JK, UFVJM, n. 5000, Rodovia MGT 367, KM 583 CEP: 39.100-000 Diamantina/MG, Brasil. Telefone: (38) 3532-1238 e-mail: apsfisio@hotmail.com 
Pereira JAC, et al. Efeito do treinamento em circuito orientado. Rev Ter Ocup Univ São Paulo. 2016 set./dez.;27(3):329-35.

\section{INTRODUÇÃO}

A doença de Parkinson (DP) requer atenção devido à avaliação sobre o aumento significativo da sua prevalência nos próximos anos ${ }^{1,2}$. É uma doença neurodegenerativa progressiva que resulta em vários sintomas motores e nãomotores. Os sintomas iniciais são causados pela atividade reduzida das células dopaminérgicas da substância nigra, adicionalmente, os circuitos motor, límbico e associativo associados aos gânglios da base são também afetados. A doença é considerada idiopática, entretanto, em alguns casos pode ser atribuída a mutação gênica ${ }^{3,4}$.

Os sintomas motores principais da DP compreendem o tremor, a bradicinesia, a rigidez e a instabilidade postural. A bradicinesia - que compromete todo o processo do movimento: planejamento, inicialização e execução - e a instabilidade postural têm um papel importante na gênese do comprometimento motor, disfunção do equilíbrio e risco de quedas. Os sintomas não-motores incluem disfunção autonômica, desordens comportamentais/cognitivas e anormalidades sensitivas e do sono ${ }^{1,2}$. Sabe-se que tanto os sintomas motores como os não-motores interferem negativamente na qualidade de vida dos pacientes ${ }^{1,2,5,6}$.

A reabilitação física tem papel importante no tratamento pois pode beneficiar aspectos motores e a qualidade de vida. Vários tipos de abordagens promovem melhorias motoras e psicológicas e o cuidado multiprofissional é considerado o modelo mais vantajoso não só para essa, mas para outras doenças neurodegenerativas ${ }^{7-9}$.

$\mathrm{O}$ treinamento em circuito orientado para tarefa (TCOT) é baseado em estações de trabalho que reproduzem atividades físicas executadas no dia-a-dia do indivíduo, ou seja, caminhar, subir escadas, manter equilíbrio, vestir-se, com o objetivo de aprendizagem motora e retenção da tarefa ${ }^{10}$. O TCOT é considerado uma boa opção de tratamento, pois além de proporcionar a prática intensiva das necessidades individuais; pode englobar dinâmicas de grupo que auxiliam na interação social que promove benefícios psicossociais, melhora do humor e da autoconfiança. Pode ter como objetivos primários a qualidade da execução, o tempo de execução ou a progressão das atividades; além disso, os pacientes, talvez pela vontade de se superarem, ficam mais engajados com a terapia em comparação aos tratamentos tradicionais ${ }^{11}$. Os efeitos do TCOT foram descritos para pacientes com acidente vascular cerebral (AVC) e com esclerose múltipla $(\mathrm{EM})^{10,11}$, não há descrições para pacientes com DP. Desta forma, este estudo apresenta a descrição de um modelo de TCOT para estes pacientes.

\section{PROCEDIMENTOS METODOLÓGICOS}

A pesquisa foi submetida e aprovada pelo Comitê de Ética em Pesquisa da Universidade Federal dos Vales do Jequitinhonha e Mucuri (UFVJM), protocolo número: 14502113.2.0000.5108, 07/04/2014. Participaram deste estudo dois homens, M.P.S. de 67 anos e T.A.M. de 73 anos, com diagnóstico de DP que preencheram os critérios de inclusão: diagnóstico médico de DP, aceitar participar da pesquisa, ausência de doenças associadas, de incapacidade grave (estágios 4 e 5 na Escala de Hoehn e Yahr Modificada) ${ }^{12}$ e de prática de qualquer atividade física e de reabilitação.

O estudo foi realizado na Clínica Escola de Fisioterapia da UFVJM, durante seis semanas, com duas sessões semanais, totalizando 12 sessões de TCOT. Os pacientes passaram por avaliação antes e após o TCOT. Foram realizados o teste Romberg sensibilizado ${ }^{13}-\mathrm{o}$ paciente deveria colocar o calcâneo de um pé em frente ao hálux do outro pé e permanecer por 30 segundos de olhos fechados -; o teste de velocidade da marcha ${ }^{14}$ - o paciente foi instruído a caminhar o mais rápido que conseguisse em um corredor de solo estável e seguro, previamente marcado com fitas indicando o comprimento total de 14 metros. Os dois metros iniciais e finais não foram registrados (momentos de aceleração e desaceleração); e o teste Timed Up \& Go (TUG) ${ }^{15}$ - o paciente foi instruído a levantar de uma cadeira, caminhar 3 metros em velocidade habitual com seus calçados usuais, virar-se e retornar sentando novamente na cadeira. Os pacientes responderam ao Questionário da doença de Parkinson (PDQ-39) que compreende 39 itens, divididos em oito categorias: mobilidade, atividades de vida diária, bem-estar emocional, estigma, apoio social, cognição, comunicação e desconforto corporal. A pontuação varia de 0 a 100 e quanto menor a pontuação melhor a percepção do indivíduo sobre sua qualidade de vida $^{12}$. Além disso, foram avaliados pela Escala Unificada para a Avaliação da Doença de Parkinson (UPDRS), que além dos sintomas avalia as incapacidades da doença. É composta por 42 itens e considera aspectos relacionados a atividade mental; comportamento e humor; atividades de vida diária; exploração motora e complicações da terapia medicamentosa. Quanto maior for a pontuação, maior o comprometimento pela doença $\mathrm{a}^{16}$.

O circuito foi composto por cinco estações com diferentes tarefas específicas a serem cumpridas, os pacientes foram instruídos para executá-las evitando pausas. O tempo em cada estação durante todo o protocolo 
foi registrado, e o tempo total de execução do circuito a cada dia somado. Os pacientes realizaram as 12 sessões sempre no período vespertino, e no estado "on". As estações foram:

- Equilíbrio e marcha: foram utilizados bastões (quatro $\mathrm{cm}$ de altura), colchonetes (cinco $\mathrm{cm}$ de altura), e a escada de canto com rampa para formar um caminho com obstáculos e superfícies irregulares no qual o paciente tinha que realizar a ida e a volta (Figura 1A);

- Destreza: foi utilizado um labirinto elétrico constituído por um arame rodeado por uma argola, o paciente deveria movimentar a argola durante o percurso do arame sem que os dois objetos se tocassem, se isso ocorresse uma luz vermelha se acendia e o número de erros pôde dessa forma ser registrado. O paciente realizava o percurso com a mão direita e retornava com a mão esquerda (Figura 1B). Logo em seguida, o paciente tinha que desenhar o espiral de Arquimedes, da melhor forma possível, sem que as linhas se tocassem (Figura 1C);
- Bradicinesia e equilíbrio: foram utilizados exercícios de arremesso de bolas com pesos diferentes (uma bola de plástico leve e uma bola de $1 \mathrm{~kg}$ ) entre o paciente e a terapeuta em seis direções (esquerda, direita e centro, na altura da cabeça e na altura da joelho). A distância entre o paciente e a terapeuta foi de três metros (Figura 1D).

- Função membros superiores: O paciente foi solicitado a retirar um jaleco do cabide, vesti-lo, abotoar os quatro botões, desabotoá-lo e colocá-lo novamente aberto no cabide (Figura 1E);

- Função membros inferiores: O paciente foi solicitado a sentar e levantar de uma cadeira durante 10 vezes consecutivas e sem a utilização dos membros superiores como apoio (Figura 1F) e em seguida percorrer o mais rápido possível um trajeto de 10 metros (Figura 1G).

Foi realizada análise descritiva dos dados e estes aparecem em média, desvio padrão e porcentagem.
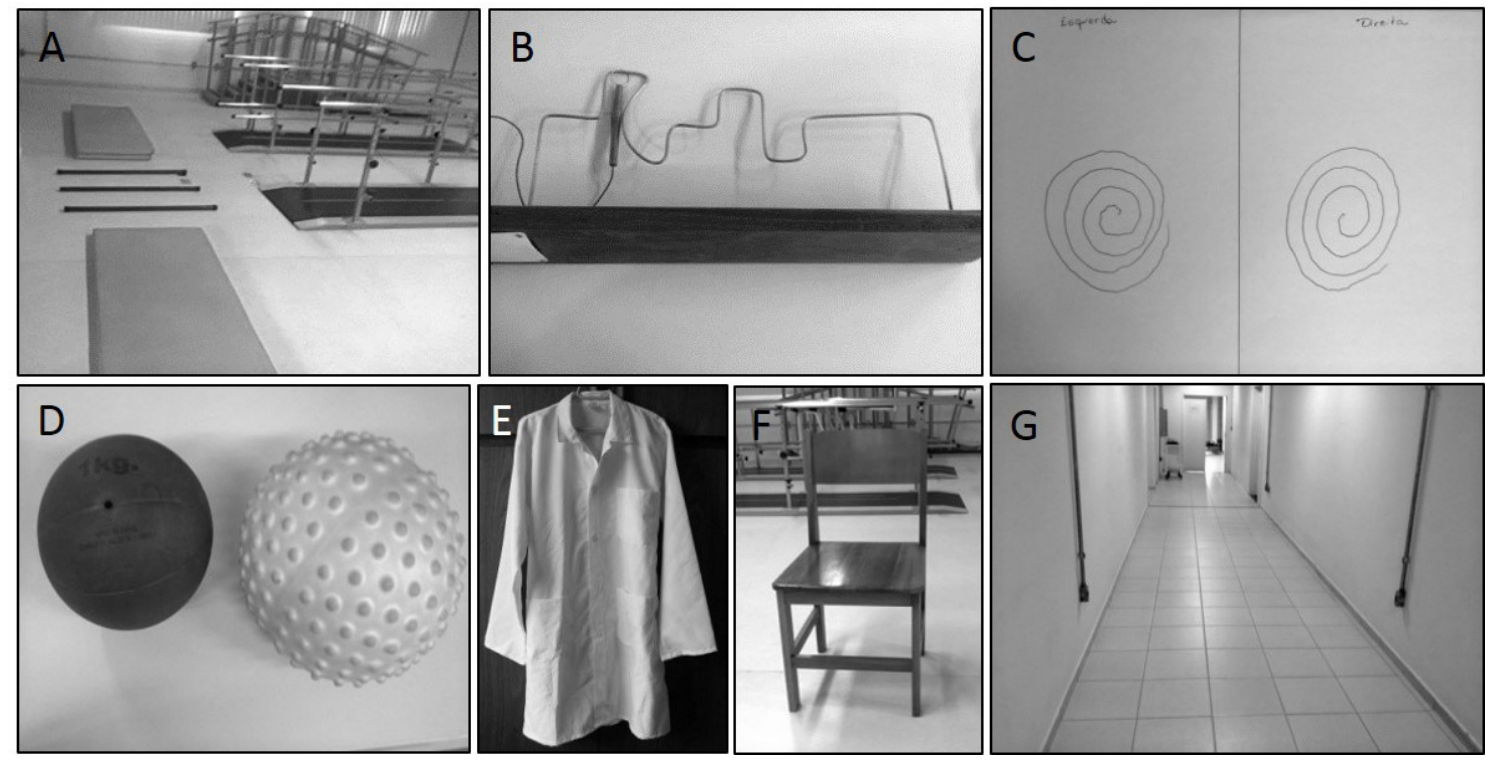

Figura 1 - Estações propostas para o TCOT: A - dispositivos utilizados na estação 1; B - labirinto elétrico; C espiral de Arquimedes desenhado por um dos pacientes; D; E; F e G - bolas; jaleco; cadeira e corredor utilizados nas estações 3, 4 e 5, respectivamente 
Pereira JAC, et al. Efeito do treinamento em circuito orientado. Rev Ter Ocup Univ São Paulo. 2016 set./dez.;27(3):329-35.

\section{RESULTADOS}

Os dados referentes às avaliações realizadas estão evidenciados nas tabelas 1 e 2 . Ambos os pacientes apresentaram melhora no desempenho (qualidade da execução e velocidade) de todas as estações do circuito durante o período de treinamento. Os erros do paciente M.P.S. na estação 2a diminuíram de 58 no primeiro dia para 19 no último dia. Já o paciente T.A.M. de 27 erros registrados no primeiro dia passou para oito no último dia de treinamento. $\mathrm{O}$ tempo de execução das tarefas está discriminado na figuras 2 e 3 para os pacientes M.P.S. e T.A.M., respectivamente.

Tabela 1 - Resultados dos testes aplicados antes e após o TCOT

\begin{tabular}{l|c|c|c|c|c|c}
\hline \multirow{2}{*}{ Pacientes } & Avaliação & $\begin{array}{c}\text { Romberg } \\
\text { Sensibilizado }\end{array}$ & VM & TUG & UPDRS & TE do circuito \\
\hline \multirow{2}{*}{ M.P.S. } & Inicial & $30 \mathrm{~s} \mathrm{com} \mathrm{I}$ & $1,38 \mathrm{~m} / \mathrm{s}$ & $9,28 \mathrm{~s}$ & 12 & $7 \mathrm{~min} 15 \mathrm{~s}$ \\
\cline { 2 - 8 } & Final & $30 \mathrm{~s} \mathrm{sem} \mathrm{I}$ & $1,82 \mathrm{~m} / \mathrm{s}$ & $8,17 \mathrm{~s}$ & 7 & $4 \mathrm{~min} 48 \mathrm{~s}$ \\
\hline \multirow{2}{*}{ T.A.M. } & Inicial & $0 \mathrm{~s}$ & $1,15 \mathrm{~m} / \mathrm{s}$ & $10,47 \mathrm{~s}$ & 35 & $9 \mathrm{~min} 7 \mathrm{~s}$ \\
\cline { 2 - 8 } & Final & $10 \mathrm{~s}$ & $1,29 \mathrm{~m} / \mathrm{s}$ & $9,27 \mathrm{~s}$ & 20 & $6 \mathrm{~min} 11 \mathrm{~s}$ \\
\hline
\end{tabular}

Legenda: I - instabilidade (oscilações de tronco e membros superiores); VM - velocidade da marcha em metros por segundo; TUG - Timed Up \& Go em segundos (sendo considerando um resultado normal para adultos independentes um valor menor do que 10 segundos ${ }^{17}$ ); UPDRS - Escala Unificada para a Avaliação da Doença de Parkinson; TE - tempo de execução do circuito em minutos e segundos

Tabela 2 - Escores por área do PDQ-39 obtidos pelos pacientes

\begin{tabular}{|c|c|c|c|c|}
\hline \multirow[t]{2}{*}{ Áreas } & \multicolumn{2}{|c|}{$\begin{array}{c}\text { Escore - Avaliação Inicial } \\
(0 \text { a 100\%) }\end{array}$} & \multicolumn{2}{|c|}{$\begin{array}{c}\text { Escore - Avaliação Final } \\
(0 \text { a 100\%) }\end{array}$} \\
\hline & M.P.S. & T.A.M. & M.P.S. & T.A.M. \\
\hline Mobilidade & $10 \%$ & $90 \%$ & 0 & $32,5 \%$ \\
\hline AVD & 0 & $83,33 \%$ & 0 & $41,66 \%$ \\
\hline Bem estar emocional & $8,33 \%$ & $12,5 \%$ & 0 & 0 \\
\hline Estigma & 0 & $37,5 \%$ & 0 & 0 \\
\hline Suporte social & 0 & $50 \%$ & 0 & 0 \\
\hline Cognição & $18,75 \%$ & $75 \%$ & 0 & $12,5 \%$ \\
\hline Comunicação & 0 & $8,33 \%$ & 0 & $16,66 \%$ \\
\hline Desconforto corporal & $8,33 \%$ & $41,67 \%$ & $8,33 \%$ & $16,66 \%$ \\
\hline
\end{tabular}

Legenda: A pontuação é caracterizada pela porcentagem obtida pelo paciente, quanto maior a porcentagem de cada área, pior a percepção do paciente quanto à sua qualidade de vida. AVD: atividade de vida diária

\section{Tempo de Execução das Estações}

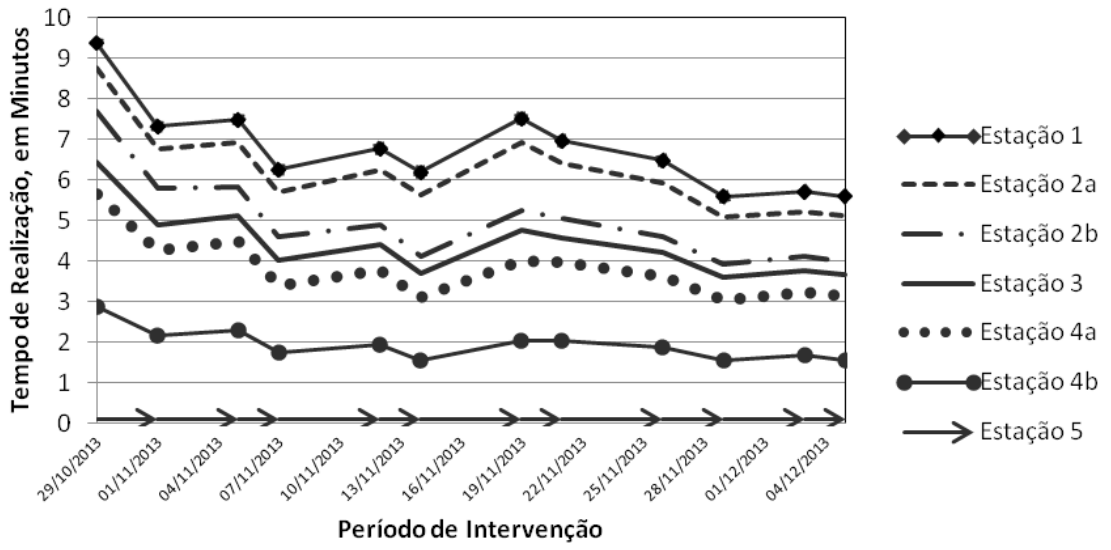

Figura 2 - Tempo de execução das estações do treinamento em circuito orientado para tarefa para o paciente M.P.S 


\section{Tempo de Execução das Estações}

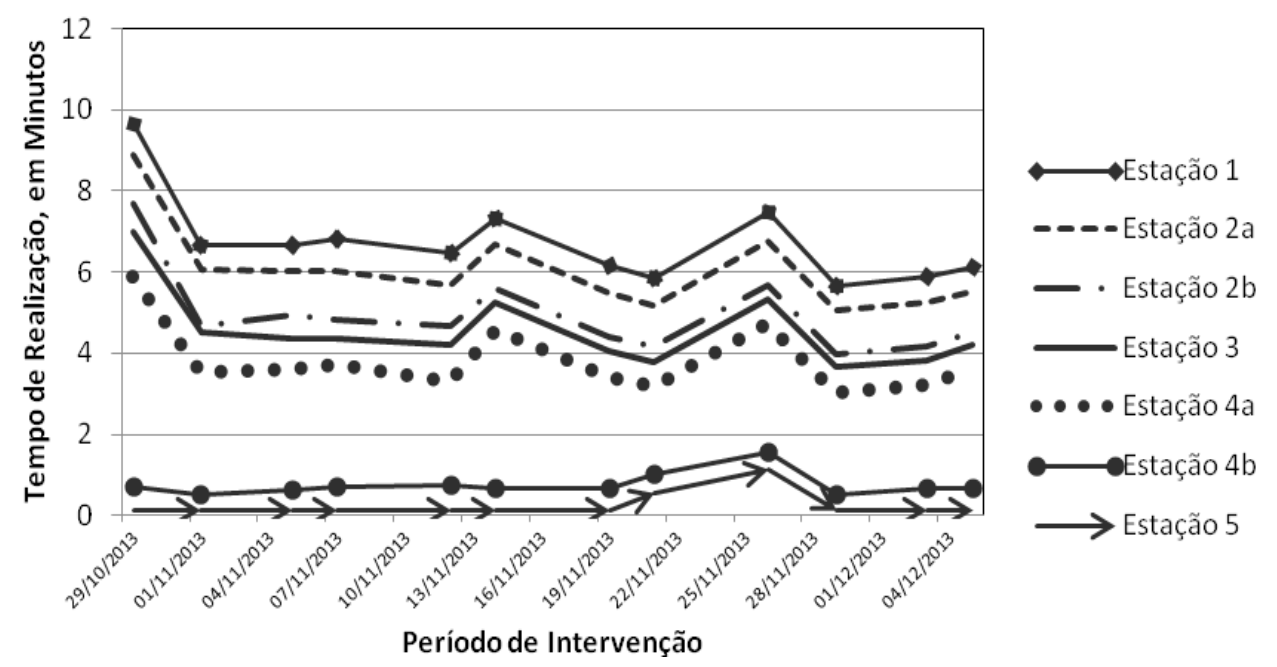

Figura 3 - Tempo de execução das estações do treinamento em circuito orientado para tarefa para o paciente T.A.M

\section{DISCUSSÃO}

O TCOT foi uma abordagem útil para os aspectos funcionais e emocionais dos dois indivíduos avaliados e não foram encontrados estudos específicos sobre este modelo de reabilitação na DP. Entretanto, há descrição da inclusão de exercícios orientados à tarefa, dentro de um programa de reabilitação multiprofissional voltado a pacientes com $\mathrm{DP}^{17}$. Este estudo, controlado e randomizado, evidenciou que um programa utilizando exercícios orientados à tarefa associados a treinamento cognitivo e educação ergonômica conduzidos por psicólogo, terapeuta ocupacional e fisioterapeuta é superior a sessões apenas de fisioterapia. Além disso, pontua o possível efeito dos exercícios orientados à tarefa como os responsáveis pela melhoria da instabilidade postural e do risco de quedas. Essa melhoria pode ser observada nos dois casos aqui estudados onde a resposta ao teste Romberg, ao TUG e à velocidade da marcha foram melhores após o TCOT proposto. Estes testes foram escolhidos, pois além de válidos, são fáceis e rápidos para se executar nos mais diversos contextos institucionais e comunitários, requisitos importantes a serem considerados pelos profissionais da saúde que precisam de medidas clínicas confiáveis para avaliar seus pacientes ${ }^{13-15,18}$. O TUG além de ser útil na avaliação da mobilidade, equilíbrio, risco de quedas e dependência para as atividades de vida diária é adequado para quantificar distúrbios que resultam no escasso sequenciamento das habilidades motoras aprendidas e indicado para pacientes com DP com incapacidade leve e moderada $^{18,19}$. A velocidade da marcha e o teste Romberg, da mesma forma, são parâmetros adequados para avaliar alterações do equilíbrio desta população. Aquela ainda é associada à diminuição das funções executivas nos indivíduos com $\mathrm{DP}^{20,21}$.

Houve melhoria na qualidade e na velocidade da execução das tarefas com o TCOT. Resultado esperado em uma reabilitação que envolve aprendizado motor e retenção de tarefa e muito importante para pacientes que têm a bradicinesia como sintoma cardinal ${ }^{2,10}$. A melhora do escore total da UPDRS e da qualidade de vida dos dois pacientes foi perceptível, esta com melhorias em todas as áreas exceto comunicação para o paciente T.A.M. e desconforto corporal para o M.P.S. As incapacidades funcionais, a instabilidade postural, o prejuízo cognitivo e a depressão são os fatores que mais influenciam a qualidade de vida em indivíduos com $\mathrm{DP}^{22}$. Dessa forma, o TCOT pode ser uma interessante ferramenta para influenciar de forma positiva a qualidade de vida do indivíduo com DP, pois é uma abordagem que permite desenvolver um trabalho relacionado a todos estes fatores.

Algumas vantagens do TCOT têm que ser consideradas: é uma atividade que pode ser realizada individualmente ou em grupo; pode ser dinâmica, versátil, desafiadora e de fácil aplicação; apresenta uma ampla variedade de possibilidades de objetivos, dispositivos, tarefas e ambientes; pode ser utilizada como uma 
abordagem para as mais diversas doenças e disfunções de forma não só reabilitadora, mas preventiva; e somado a tudo isso pode ser praticada por diversos profissionais que trabalham com reabilitação em todos os níveis de atenção à saúde de forma individualizada ou integrada. A dinâmica entre as estações do circuito: executar cada tarefa uma após a outra, com o mínimo de intervalo entre elas e verificando o tempo de execução em cada estação e o geral, pode ser uma boa opção para pacientes com DP que além da lentidão característica, apresentam com frequência dificuldade em iniciar os movimentos e de realizar tarefas de forma sequencial. Além disso, alguns pacientes com DP apresentam freezing que acomete principalmente os membros inferiores e pode ser manifestado pela incapacidade súbita e transitória para se mover, podendo incluir desde uma hesitação inicial a uma súbita incapacidade para mover os pés em situações específicas como, por exemplo, aproximar-se do seu destino ou passar por um local estreito3, situações estas que o circuito oferece e precisam ser enfrentadas. Ademais, a ferramenta tempo que o circuito oferece, além de estímulo para o paciente, acaba por ser um parâmetro de análise para o terapeuta.

Os estudos encontrados sobre o TCOT em pacientes com AVC e EM tiveram resultados positivos. Pacientes crônicos com AVC obtiveram melhora da marcha e de atividades relacionadas a marcha11 e pacientes com EM obtiveram melhora da função motora e da qualidade de vida10,23, assim como o encontrado neste estudo para pacientes com DP. Além do TCOT, ressalta-se que estudos envolvendo atividade de reabilitação ou física no formato de circuito, porém, direcionados a outros objetivos são encontrados na literatura. Houve melhora da força muscular e resistência associada com significativa redução da dor no ombro durante as atividades diárias em paraplégicos após um programa de resistência em circuito ${ }^{24}$. Ademais, os benefícios da atividade em circuito para a população idosa têm sido demonstrados ${ }^{25}$.

A descrição dos efeitos do TCOT em dois pacientes com DP torna-se importante pela escassez deste assunto na literatura, pela prevalência atual de casos e que se tornará mais significativa com o passar dos anos, pelas características específicas que dificultam a realização de atividades diárias e, consequentemente, ocasionam um declínio na funcionalidade ${ }^{5,10}$, mas a descrição destes efeitos em apenas dois pacientes é uma limitação do estudo. O número de pacientes não foi maior, pois o estudo foi direcionado exclusivamente aos pacientes com DP sem qualquer doença associada, sem incapacidade grave e que não participasse de qualquer tipo de intervenção física, de reabilitação e alternativas para que os resultados pudessem evidenciar o real papel do TCOT. Novas propostas de TCOT serão analisadas para os pacientes com DP, como por exemplo, a adição de estímulos sonoros rítmicos, para verificar se há melhora dos resultados obtidos já que os mesmos se relacionam com melhora da mobilidade e do risco de quedas nestes pacientes $^{26}$.

\section{CONCLUSÃO}

Um programa de TCOT, constituído por cinco estações contendo propostas direcionadas para funcionalidade, equilíbrio e destreza manual, proporcionou melhora no desempenho motor e na qualidade de vida dos dois pacientes com DP.

\section{REFERÊNCIAS}

1. Farag I, Sherrington C, Hayes A, Canning CG, Lord SR, Close $\mathrm{JC}$, et al. Economic evaluation of a falls prevention exercise program among people with Parkinson's disease. Mov Disord. 2015:1-9 [Epub ahead of print] DOI: 10.1002/mds.26420.

2. Ferguson LW, Rajput AH, Rajput A. Early-onset vs. lateonset Parkinson's disease: A clinical-pathological study. Can J Neurol Sci. 2015;20:1-7 [Epub ahead of print] DOI: 10.1017/cjn.2015.244.

3. Jankovic J. Parkinson's disease: clinical features and diagnosis. J Neurol Neurosurg Psychiatr. 2008;79(4):368-76. DOI: 10.1136/jnnp.2007.131045.
4. Sumec R, Filip P, Sheardová K, Bares M. Psychological benefits of nonpharmacological methods aimed for improving balance in Parkinson's disease: a systematic review. Behav Neurol. 2015;1-16 [Epub ahead of print] DOI: $10.1155 / 2015 / 620674$.

5. Schrag A, Jahanshahi M, Quinn N. What contributes to quality of life in patients with Parkinson's disease? J Neurol Neurosurg Psychiatr. 2000;69:308-12. DOI 10.1136/ jnnp.69.3.308.

6. Canon MBF, Novelli MMPC. Identificação dos sintomas comportamentais e psicológicos em idosos moradores de uma 
Instituição de Longa Permanência. Rev Ter Ocup Univ São Paulo. 2012;23(1):72-80. DOI: http://dx.doi.org/10.11606/ issn.2238-6149.v23i1p72-80.

7. Goodwin VA, Richards SH, Taylor RS, Taylor AH, Campbell JL. The effectiveness of exercise interventions for people with parkinson's disease: a systematic review and metaanalysis. Mov Disord. 2008;23(5):631-40. DOI: 10.1002/ mds.21922.

8. Cruickshank TM, Thompson JA, Domínguez D JF, Reyes AP, Bynevelt M, Georgiou-Karistianis N, et al. The effect of multidisciplinary rehabilitation on brain structure and cognition in Huntington's disease: an exploratory study. Brain Behav. 2015;5(2):1-10. DOI: 10.1002/brb3.312.

9. Van der Marck MA, Bloem BR, Borm GF, Overeem S, Munneke M, Guttman M. Effectiveness of multidisciplinary care for Parkinson's disease: a randomized, controlled trial. Mov Disord. 2013;28(5):605-11. DOI: 10.1002/mds.25194.

10. Straudi S, Martinuzzi C, Pavarelli C, Sabbagh Charabati A, Benedetti $\mathrm{MG}$, Foti $\mathrm{C}$, et al. A task-oriented circuit training in multiple sclerosis: a feasibility study. BMC Neurol. 2014;7(14):1-9. DOI: 10.1186/1471-2377-14-124.

11. Wevers L, vandePortI,VermueM,MeadG,KwakkelG.Effects of task-oriented circuit class training on walking competency after stroke: a systematic review. Stroke. 2009;40(7):2450-9. DOI: 10.1161/STROKEAHA.108.541946.

12. Tiago MSF, Almeida FO, Santos LS, Veronezi RJB. Instrumentos de avaliação de qualidade de vida na doença de Parkinson. Rev Neurocienc. 2010;18(4):538-43.

13. Weber PC, Cass SP. Clinical assessment of postural stability. Am J Otol. 1993;14(6):566-9.

14. Salbach NM, Mayo NE, Higgins J, Ahmed S, Finch LE, Richards CL. Responsiveness and predictability of gait speed and other disability measures in acute stroke. Arch Phys Med Rehabil. 2001;82(9):1204-12. DOI: http://dx.doi. org/10.1053/apmr.2001.24907.

15. Podsiadlo D, Richardson S. The timed "Up \& Go": a test of basic functional mobility for frail elderly persons. J Am Geriatr Soc. 1991;39:142-8.

16. Fahn S, Elton RL, and members of the UPDRS Development Committee. Unified Parkinson's Disease Rating Scale. In: Fahn S, Marsden CD, Calne D, Goldstein M. Recent developments in Parkinson's disease. Florham Park [NJ, USA]: Macmillan Healthcare Information; 1987. p.153-63.
17. Monticone M, Ambrosini E, Laurini A, Rocca B, Foti C. In-patient multidisciplinary rehabilitation for Parkinson's disease: a randomized controlled trial. Mov Disord. 2015;30(8):1050-8. DOI: 10.1002/mds.26256.

18. Morris S, Morris ME, Iansek R. Reliability of measurements obtained with the Timed "Up \& Go" test in people with Parkinson disease. Phys Ther. 2001;81(2):810-8.

19. Cândido DP, Cillo BAL, Fernandes AS, Nalesso RP, Jakaitis F, Santos DG. Análise dos efeitos da dupla tarefa na marcha de pacientes com doença de Parkinson: relato de três casos. Rev Neurocienc. 2012;20(2):240-5.

20. Stegemöller EL, Wilson JP, Hazamy A, Shelley MC, Okun MS, Altmann LJ, Hass CJ. Associations between cognitive and gait performance during single- and dual-task walking in people with Parkinson disease. Phys Ther. 2014;94(6):75766. DOI: 10.2522/ptj.20130251.

21 Ickenstein GW, Ambach H, Klöditz A, Koch H, Isenmann S, Reichmann H, Ziemssen T. Static posturography in aging and Parkinson's disease. Front Aging Neurosci. 2012;4(20):1-7. DOI: 10.3389/fnagi.2012.00020.

22. Schrag A, Jahanshahi M, Quinn N. What contributes to quality of life in patients with Parkinson's disease? J Neurol Neurosurg Psychiatry. 2000;69(3):308-12. DOI: 10.1136/jnnp.69.3.308.

23. Chisari C, Venturi M, Bertolucci F, Fanciullacci C, Rossi B. Benefits of an intensive task-oriented circuit training in multiple sclerosis patients with mild disability. NeuroRehabilitation. 2014;35(3):509-18. DOI: 10.3233/NRE-141144.

24. Nash MS; van de Vem I; van ElkN; Johnson BM. Effects of circuit resistance training on fitness attributes and upper-extremity pain in middle-aged men with paraplegia. Arch Phys Med Rehabil. 2007;88(1):70-5. DOI: 10.1016/j.apmr.2006.10.003.

25. Arenas SR; Pascual MM; Alcaraz PE. Impact of resistance circuit training on neuromuscular, cardiorespiratory and body composition adaptations in the elderly. Aging Dis. 2013;4(5):256-63. DOI: 10.14336/AD.2013.0400256.

26. Hausdorff JM, Lowenthal J, Herman T, Gruendlinger L, Peretz C, Giladi N. Rhythmic auditory stimulation modulates gait variability in Parkinson's disease. Eur J Neurosci. 2007;26(8):2369-75. DOI:10.1111/j.1460-9568.2007.05810.x

Recebido em: 04.10.15

Aceito em: 17.06.16 